\title{
Robust video communication over an urban VANET
}

\author{
N. Qadri, M. Altaf, M. Fleury* and M. Ghanbari \\ School of Computer Science and Electronic Engineering, University of Essex, Wivenhoe Park, \\ Colchester, CO4 3SQ, UK
}

\begin{abstract}
Video communication within a Vehicular Ad Hoc Network (VANET) has the potential to be of considerable benefit in an urban emergency, as it allows emergency vehicles approaching the scene to better understand the nature of the emergency. However, the lack of centralized routing and network resource management within a VANET is an impediment to video streaming. To overcome these problems the paper pioneers source-coding techniques for VANET video streaming. The paper firstly investigates two practical multiple-path schemes, Video Redundancy Coding (VRC) and the H.264/AVC codec's redundant frames. The VRC scheme is reinforced by gradual decoder refresh to improve the delivered video quality. Evaluation shows that multiple-path 'redundant frames' achieves acceptable video quality at some destinations, whereas VRC is insufficient. The paper also demonstrates a third source coding scheme, single-path streaming with Flexible Macroblock Ordering, which is also capable of delivery of reasonable quality video. Therefore, video communication between vehicles is indeed shown to be feasible in an urban emergency if the suitable source coding techniques are selected.
\end{abstract}

Keywords: Error resilience, IEEE 802.11p, multiple path delivery, redundant frames, VANET, video communication

\section{Introduction}

This paper considers how to support robust video communication across multi-hop networks between vehicles when an urban emergency occurs. Real-time video communication allows early responders approaching an incident [28] to better understand the nature of the problem at the scene of an emergency but the lack of centralized routing and network resource management is challenging. Crash scenes, views of fleeing vehicles or burning buildings are some illustrative applications, while there is also now a strategic incentive [28] to provide coverage during a more serious, general emergency. In all these scenarios, it is the other personnel in the emergency vehicle or passengers in the vehicle that view the arriving video stream and not the driver.

Vehicular Ad Hoc Networks (VANETs) bring several advantages to video streaming within an ad hoc network. Battery power is no longer a problem if built-in transceivers are employed, implying that larger buffers (with passive and active energy consumption) can now serve to absorb any latency arising from multi-hop routing. We consider urban VANETs. Within a city, because of traffic congestion, high speeds do not generally arise. Therefore, connections are on average longer and Doppler effects are limited. Vehicle motion is indeed restricted by the road geometry but compared to a highway VANET vehicle motion is no longer linear.

\footnotetext{
${ }^{*}$ Corresponding author. Tel.: +44 1206 872817; Fax: +44 1206 872900; E-mail: fleum@essex.ac.uk.
} 
We examine three alternative video practical source-coding schemes for emergency video streaming, with one of the schemes applied in two different ways. The source-coding techniques applied exist in the context of IP networking [39] but, as far as the authors are aware, they have not been applied elsewhere in the way described within a VANET context. The first scheme examined is a variant of Multiple Description Coding (MDC) [36] in which two or more versions or descriptions of the same video stream are sent over different, preferably disjoint, routes across a network. Either description can serve to reconstruct the video but enhanced quality is produced by combining both descriptions. If adverse conditions occur on one of the paths then the packetized encoded bitstream from the other path can compensate. Video Redundancy Coding (VRC) [40] is the simplified MDC scheme employed by us that in the event of packet loss does not require additional decoder reconciliation between the two descriptions. Additionally, the VRC scheme was also trialed using distributed intra-coded macroblocks, that is H.264/AVC (Advanced Video Coding)'s [39] Gradual Decoder Refresh (GDR) [30], to avoid the reliance on prior reference frames.

This paper also proposes a second MDC scheme, employing H.264/AVC redundant frames, which when combined with multiple-path video transfer will result in higher-quality delivered video at a cost in higher data traffic. However, this cost may well be justified in an emergency. Redundant frames $[32,42]$ (or strictly redundant slices ${ }^{1}$ making up a frame) are coarsely quantized frames that can avoid sudden drops in quality marked by freeze frame effects if a complete frame (or slice) is lost. Again assuming even and odd frames are sent separately in two streams, then redundant frames are predicted from previous frames in the same stream but do not act as a reference to later frames.

In a third scheme, our paper proposes Flexible Macroblock Ordering (FMO) [38] with Checkerboard FMO pattern for single-path video stream transfer as an alternative to multiple-path methods. Error resilience [31] is applied at a source encoder to counter potential packet loss. FMO is an error-resilient technique newly included in the H.264/AVC codec that is suitable for error-prone channels. In good channel conditions, the overhead from sending the FMO mapping is a disadvantage but this is unlikely to be a problem when multi-hop routing occurs. Through source-encoder-independent error concealment at the decoder, FMO can aid the reconstruction of frames that have lost some of their constituent packets.

To the best of the authors' knowledge, though investigation of the concept of video streaming within a VANET has occurred, source-coding techniques have not been applied to any extent to VANETs. Some of the literature that exists on this subject so far in comparison to our work is examined in Section 2. The University of California, Los Angles (UCLA) research group under the leadership of Mario Gerla has produced a range of creative ideas on VANETs, for example [12,25,28]. However, their focus is on wireless aspects and at the 2009 Wireless Days Conference they have confirmed their interest in using a variant of our FMO scheme within live vehicle convoys as a security measure. We are flattered by their interest. Finally, we should add that another apparently unique feature of this work is that we group the emergency vehicles into a multicast group to receive the video. But we also use the other vehicles in the vicinity whether emergency or not to relay the video. In this way, the efficiency of the transfer is greatly improved. This latter feature is described in Section 3 but firstly this paper reviews other investigations of video over VANETs.

\footnotetext{
${ }^{1}$ A slice is headed by a decoder resynchronisation marker and may include reversible Variable Length Decoding, aimed at countering propagation of errors arising from the sequential dependencies of entropic encoding. Consequently, a slice is a self-contained decoding unit.
} 


\section{Related research}

Earlier work on video communication over highway (not urban) VANETs [15] considered the problem of triggering remote video sources in the event of forward traffic congestion. The main problems in triggering [28] are how to reduce the number of messages reaching the remote camera(s) and how to reduce the latency in reaching those cameras (by reducing the number of hops), which is principally an issue of protocol design. In an emergency scenario as opposed to obtaining forward views of traffic congestion, it may be that video sources can be locally generated. Then an entirely different problem arises: how best to deal with heavy packet losses in the harsh urban environment. Video quality is strongly influenced by the impact of packet loss. Because successive video frames are broadly similar (except at scene cuts and changes of camera shots), to increase coding efficiency only the difference between successive frames is encoded. Consequently, at the frame level, removing temporal redundancy introduces a dependency on previously transmitted data that implies lost packets from reference frames will have an impact on future frames until a successful delivery of the next spatially-encoded anchor frame, when the decoder can be reset.

In [15], multiple vehicle video sources were modeled traveling on a 4-5 lane highway in Atlanta. Video was collected by sending from a car approaching a destination a request trigger to a camera on a remote vehicle passing that destination region. Video transport back to the requestor was by a storecarry-and-forward sub-system, though the method was not detailed in [15]. The main analysis in [15] was of delay characteristics, presumably because on a highway there should be sufficient time for the approaching vehicle to take evasive action if the forward view shows congestion or an accident.

Research in [25], extending the work in [15], simulated a two-ray wireless propagation model and imposed an application-layer Forward Error Control (FEC)-based solution through network coding. Though network coding of FEC and in particular rateless error coding is an effective means of limiting the impact of packet erasures upon streamed video, it depends on action by intervening nodes. When these nodes are not possible destinations and consequently may not be expected to make special provision for video data, then network coding is not feasible. Therefore, our paper considers alternative video protection methods that act in an end-to-end fashion, without the need for processing by intervening vehicles. Error resilience (in our schemes) is able to complement physical layer FEC, whereas when applying higher layer forms of FEC, it is better to do so in such a way that the channel code acts as an inner code to the PHY coding.

The feasibility of H.264/AVC video communication between two vehicles with IEEE $802.11 \mathrm{~b}$ transceivers in a live setting was examined in [5]. With speeds between the two vehicles on average $15 \mathrm{mph}(6.71 \mathrm{~m} / \mathrm{s})$ in a city setting (in Japan) it was reported that 'link availability' was $97.78 \%$, as opposed to on a highway at an average speed of $55 \mathrm{mps}(24.59 \mathrm{~m} / \mathrm{s})$ it was only $33.98 \%$. Average SNR was somewhat worse in the urban setting, $19.14 \mathrm{~dB}$, as opposed to $22.49 \mathrm{~dB}$ on the highway. Relative video quality was good (around 30-35 dB Peak Signal to Noise Ratio (PSNR)) and better in the city scenario with (slow-scan) rates ranging from $15-20 \mathrm{~Hz}$ and for Quarter Common Interchange Format $(\mathrm{QCIF})(176 \times 144$ pixels/frame) to CIF-resolution $(352 \times 288$ pixels/frame $)$. The test clip was the well-known 'Foreman' clip at QCIF resolution (employed in this paper also) with medium coding complexity, though 'Paris' with less temporal complexity was employed at the larger CIF resolution. The study [5] established that, for vehicles traveling in proximity to each other, video exchange is entirely feasible albeit at slow-scan rates and resolution depending on coding complexity (which is a reflection of spatial activity (within frames) and temporal activity (between frames)). Of course, these results do not necessarily translate to multi-hop video transfer. 
In a general context, the dissemination of multimedia information is a subject of active investigation within mobile systems. For example, in [21] progressive transmission of multi-resolution documents occurs so that the viewer can first view the relevance of the information before continuing with full transfer. To reduce the impact of low bandwidth capacity and delay over multi-hop connections, caching of data in the vicinity of an ad hoc node [6] is a promising approach. To support such systems in an ad hoc network, it is important that the routing protocols are optimized, for example [16] by tuning the route request flooding mechanism.

\section{VANET system}

\subsection{Emergency application}

Our system usage is captured in Fig. 1, showing an encircled crash scene. In such an urban emergency, it is envisaged that a scene is captured by one vehicle (the first emergency vehicle at the scene) that acts as the video source. Emergency vehicles now commonly carry video cameras, which in the case of the police act as a source of evidence in traffic offences. Therefore, there may be no need to capture the scene from cameras mounted on roadside masts, though these can be triggered locally as alternative video sources. Thus, either the scene can be captured manually by an emergency worker operating a vehicle mounted video camera, as already occurs when traffic police gather evidence or it can be captured through vehicle to roadside communication (vrc) or a roadside camera could be controlled by an emergency vehicle through remote communication. As how the video is captured is not central to this paper, we refer the reader to discussion of the feasibility of vrc such as in [8].

The video is distributed via WLAN-enabled vehicles to a multicast group of patrol cars, fire engines, ambulances or the like, acting as early responders to a crisis. If the multicast group consisted only of emergency vehicles (assuming less than ten responders for any one incident) then the ad hoc network size or density would be an impediment to communication. By routing the video stream over other intermediate car wireless transceivers, even though these cars are not destinations for the video stream (only the emergency vehicles are) multi-hop packet routing is more effective. Notice that though the destinations form a multicast group within the larger set of VANET-enabled vehicles, to improve robustness in all scenarios considered point-to-point communication is employed rather than a multicast protocol.

One emergency vehicle, acting as the video source, transfers the captured video by sending individual copies to each destination forming a virtual multicast group. When an MDC schemes is used each stream is split over two paths, refer to Fig. 1. Separate threads of control are able to generate these descriptions, possibly utilizing multi-core processors. It is assumed that available destinations, corresponding to other emergency vehicles in a group, are known through another emergency channel. In tests, six destinations were employed and it was found that, depending on choice of scheme, reasonable to good quality video was possible, though not for every destinations. Nevertheless, sufficient emergency vehicles would have a view of the emergency to allow preparations to be made as vehicles approached the scene.

\subsection{VANET communication}

Direct inter-vehicle communication can be an aid both to passenger comfort and to road safety [43]. Compared to a cellular network, a VANET may be toll free, avoids the delay in setting up a long communication circuit, and on a highway will operate where there are coverage gaps in a cellular 


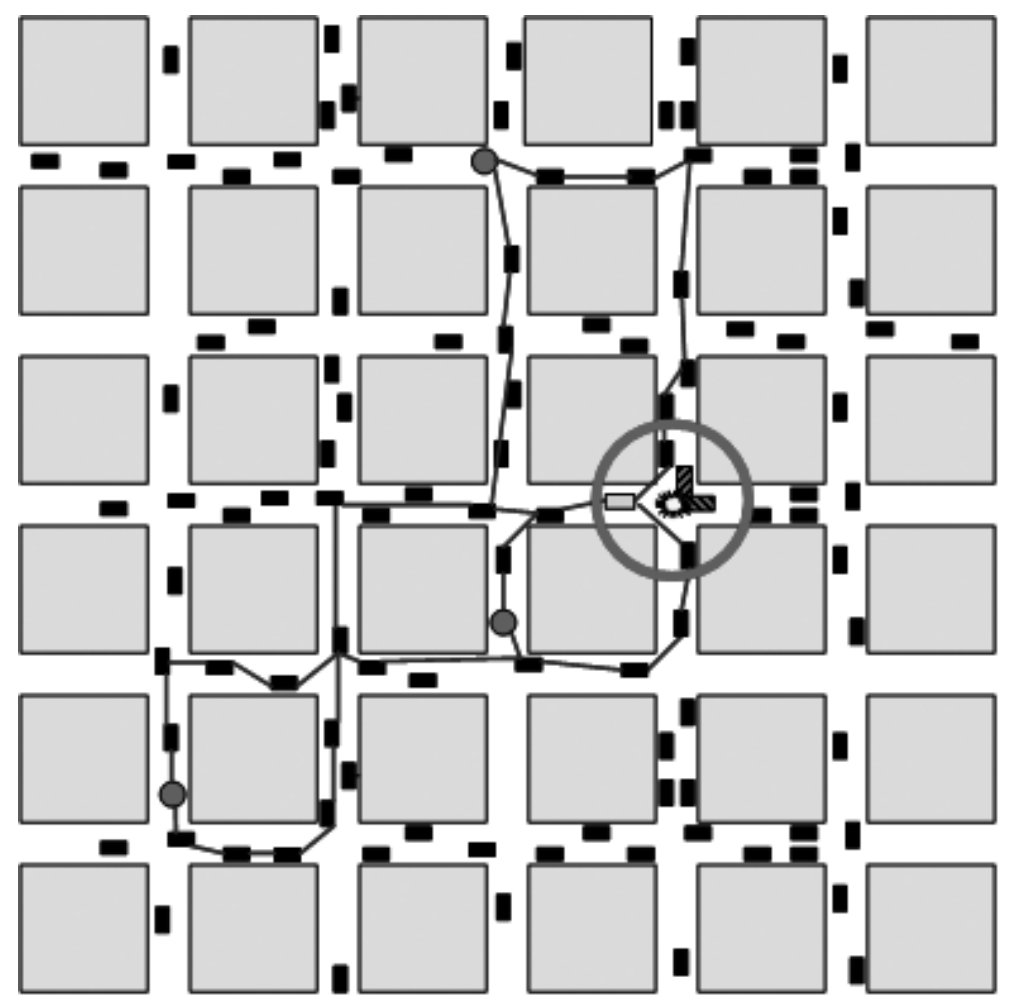

Fig. 1. VANET operating in city blocks with collision (encircled) videoed by light colored patrol car. Black rectangles are other vehicles acting as relays. Small circles are other emergency vehicles. Connecting lines show possible multi-paths for transmission of video.

network. There are strong pressures pushing car manufacturers towards equipping cars with WLAN capability, if they have not already done so. The IEEE $802.11 \mathrm{p}$ standard [17] will take advantage of 75 $\mathrm{MHz}$ of spectrum allocated both in Europe and the USA in the $5.9 \mathrm{GHz}$ range with $10 \mathrm{MHz}$ channels operating at up to $27 \mathrm{Mbps}$ depending on modulation mode. The increased safety [3] that may arise from wireless provision is under active investigation. As well as safety alerts through wayside access points, the possibility of advertising localized services provides an additional commercial incentive to wireless take-up. It is thought that early adoption will result in around 20\% of WLAN-enabled cars [4] in the near term. Therefore, at least $20 \%$ of the available cars in a city are likely to be available as relays to aid in video communication in an emergency.

If a VANET is to present an alternative to private cellular radio such as the Terrestrial Trunk Radio (TETRA) system [9] then it should provide similar services. Video communication over TETRA was explored in [7] and TETRA-2 was provided with extra bandwidth in support of multimedia communication. As an example, HW Communications Ltd. recently presented T-Serv for slow-scan video communication over TETRA, with in-vehicle video communication through IEEE 802.15.1 (Bluetooth). Compared to TETRA's cellular system, a VANET system can additionally make use of vehicles other than the emergency vehicles themselves, thus increasing coverage. (TETRA has an ad hoc mode but this is obviously confined to emergency vehicles, thus restricting the size of the ad hoc network.) Ad hoc radio is also potentially not limited by the urban 'canyons' caused by high buildings (if a base antenna is employed). 


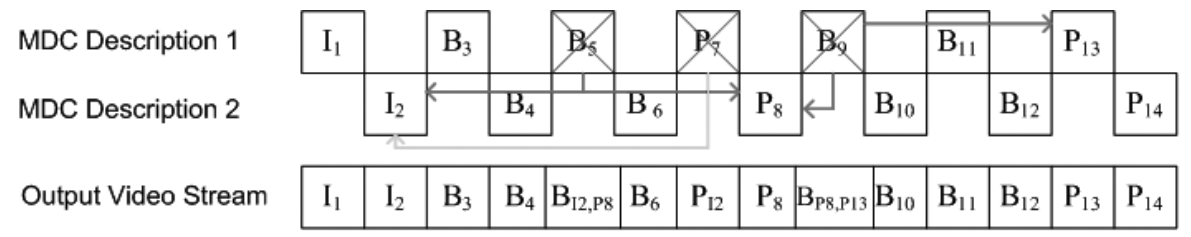

(a)

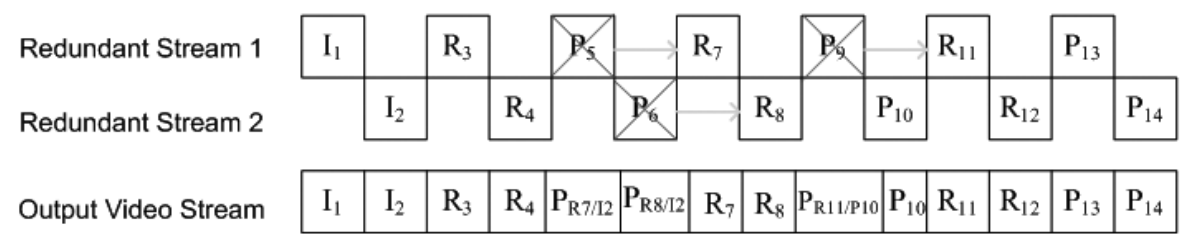

(b)

FMO Slice 0

FMO Slice 1

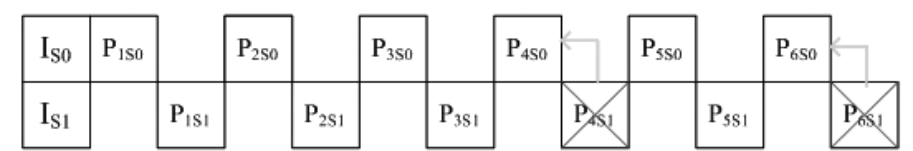

Output Stream

\begin{tabular}{|l|l|l|l|l|l|l|l|l|l|l|l|l|l|}
\hline $\mathrm{I}_{\mathrm{S} 0}$ & $\mathrm{I}_{\mathrm{SI}}$ & $\mathrm{P}_{1 \mathrm{~S} 0}$ & $\mathrm{P}_{1 \mathrm{~S} 1}$ & $\mathrm{P}_{250}$ & $\mathrm{P}_{2 \mathrm{~S} 1}$ & $\mathrm{P}_{350}$ & $\mathrm{P}_{3 \mathrm{~S} 1}$ & $\mathrm{P}_{4 \mathrm{~S} 150}$ & $\mathrm{P}_{450}$ & $\mathrm{P}_{550}$ & $\mathrm{P}_{5 \mathrm{~S} 1}$ & $\mathrm{P}_{65150}$ & $\mathrm{P}_{650}$ \\
\hline
\end{tabular}

(c)

Fig. 2. Different path diversity schemes: a) VRC with odd and even descriptions b) Two streams with redundant frames, c) FMO slice replacement scheme.

If the source and destination are both assumed to be emergency vehicles, as in the scheme presented in this paper, then, when the video is routed via non-emergency vehicles, its confidentiality can be preserved through a stream cipher or alternatively through selective encryption of compressed video [44] to reduce the computation load of full encryption. (For example, only motion vectors in the compressed steam need be encrypted, as without these it is difficult to reconstruct a video). Another approach to security [22], which requires the cooperation of intermediate nodes to perform network coding, takes advantage of disjoint paths. However, though multiple paths may be available in a VANET, it is difficult and unnecessary to ensure they are node disjoint. A further approach [12] is to allow partial disclosure of some data after a time limit has expired. However, though this scheme may reduce dissemination latency, it is not clear that it allows streaming, as it requires an all-or-nothing transform, i.e. receipt of all data, before the data can be reconstructed. Partial disclosure of content is also permitted.

\subsection{Video transfer schemes}

Figure 2 illustrates the schemes tested in this paper. The frame numbers indicate the raw video frame from which a coded frame is constructed. Frames are decoded with motion compensation from reference frames in the same stream. By separately decoding from each stream, the problem of MDC decoder complexity is avoided. Figure 2a shows a number of frames have been dropped (marked by crossing out). Lost frames in one description can be reconstructed by reference to other correctly received packets in either description, with arrowed lines in Fig. 2a indicating the reconstruction route. For example, $\mathrm{B}_{5}$ has been lost in description 1 and is reconstructed from either $\mathrm{I}_{2}$ and $\mathrm{P}_{8}$. The final row of frames in Fig. 2a shows the frame display sequence arising after substitution of reconstructed frames. Not shown in Fig. 2a is the VRC variant in which IDR-frames are no longer included in the sequence (refer to Section 4). 
In Fig. 2b, showing the redundant frame scheme, the absence of B-frames allows use of the computationally efficient H.264/AVC Baseline profile. Redundant frames are sent in each stream, at a potential cost in latency but a potential gain in delivered video quality. There is only one initial IDR-frame in each sequence, which can be replaced by an intra-coded redundant frame. All other redundant frames are normally encoded in inter-mode (with the same reference as the frame that they back up) with normally a coarser quantization setting than the frame they back-up. Again example packet losses are shown and the result of reconstructing the sequences appears in the final row of Fig. $2 \mathrm{~b}$. For example, $\mathrm{P}_{5}$ has been lost and reconstructed from $\mathrm{R}_{7}$ but if $\mathrm{R}_{7}$ did not survive it could also have been reconstructed from $\mathrm{I}_{2}$. Figure $2 \mathrm{c}$ shows the FMO method of error resilience in which each frame has been split into two slice groups. That is each frame is divided into two slices and sent in different packets. The packets are multiplexed onto a single stream with slice 0's packet preceding slice 1's packet (though the order is not important). When a packet bearing one of a frame's slices is lost then the corresponding slice is normally employed to reconstruct it through the non-normative error concealment procedure. If both slices are lost then previous frame replacement is reverted to. The final row now shows the frame receiving order from a single stream.

\section{Proposed schemes}

The first scheme proposed for use in the VANET emergency is VRC [40], which avoids the need for decoder reconciliation in the event of packet loss. This is because two independent streams are formed from separately encoded odd and even frame sequences. Some lack of coding efficiency occurs as the motion between frames in any one description is likely to be greater than if the frames were coded in their original order. Consequently, the residual or difference data, which is actually coded by predictive coding, has a larger dynamic range requiring more bits to code. By insertion of IDR-frames in both descriptions (streams), the descriptions can be resynchronized even if one of the IDR frames is lost, at a cost in increased data redundancy compared to sending a single set of IDR-frames in a single stream. The macroblocks of IDR frames are completely spatially- (intra-) coded without removal of temporal redundancy and, therefore, do not reference any other frames. Consequently, they act as anchor frames for predictively- (inter-) coded subsequent frames. (In a hybrid video codec, each frame is split into macroblocks for processing purposes. Further details can be found in a textbook such as [13].)

In compensation, for the reduction in coding efficiency resulting from employing IDR-frames, bipredictive B-frames are included within each Group of Pictures (GOP) within a VRC description to improve efficiency through multiple references (with a 10\% bit-rate reduction in H.264/AVC [29]). (Notice that in the H.264/Advanced Video Codec (AVC) [41], IDR frames prevent predictive reference across GOP boundaries, whereas H.264/AVC I-frames do not (unlike their usage in earlier codecs)). Either an IDR- or an I-frame is inserted after every 12 or 15 frames making up a GOP.

IDR frames cause periodic increases in the data rate and consequently introduce additional buffering delay. Therefore, as an alternative form of VRC, we also distributed an equivalent number of intra-coded macroblocks [30] to those contained in the IDR-frames across the two VRC descriptions. Though another function of IDR-frame insertion is to provide a random access facility (supporting video player functions), this is unlikely to be required in an emergency scenario. In this variant of VRC apart from initial IDR-frames in each description only predictively-coded P-frames occur. This results in some loss of coding efficiency but improves computational efficiency (as the need to conduct more than one 
predictive search for B-frames is no longer needed) ${ }^{2}$. Along with Constant Bit Rate (CBR) encoding, an all $\mathrm{P}$-frame sequence reduces delay for real-time applications. The risk of continued error propagation from the loss of any one IDR-frame-bearing packet is also reduced by distributing intra-macroblocks across all frames, H.264/AVC's GDR [39].

In general, MDC is computationally complex and requires specialist codecs [36], because synchronization between encoder and decoder is necessary to reduce motion estimation error drift. In a two stream MDC scheme, synchronization normally requires a third decoder in addition to the decoders that produce the reduced quality streams from single descriptions [14]. VRC is a simplified version of MDC with only two decoders, the output of which is interleaved before display. As mentioned previously, VRC normally requires the inclusion of IDR frames to allow decoder reset in the event of packet loss. If the packets happen to belong to an IDR frame then an IDR frame in the other sequence can serve as an anchor.

To avoid the need to send IDR frames, in Multiple State Video Coding (MSVC) [1] lost frames in one description are reconstructed from temporally adjacent frames in the other description. In this solution, all frames apart from the first IDR frame in each description are P-frames and reconstruction may also occur with the aid of past and future P-frames. However, reconstruction with P-frames from a different description reintroduces the risk of picture drift from the lack of synchronization between an encoder and decoder. For that reason MSVC is not tested in this paper, though MSVC can be credited as the basis of several later practical MDC schemes.

To overcome picture drift, redundant frames intended for error resilience in H.264/AVC [32,42], can serve to better reconstruct $\mathrm{P}$-frames received in error. Though redundant frames were originally intended for Internet video streaming, applying redundant frames to multiple path streaming over general ad hoc network video was independently investigated in [26,33]. However, in [26] redundant pictures in one stream were encoded based on primary frames (frames for which redundant representations are generated) in a second stream, which requires modification of the operation of the H.264 codec. Another multiple path version [33] combines slicing with redundant data. In this version, a frame is split between alternate slices (formed from macroblock rows). Each slice is either a primary slice or a redundant slice for the matching primary slice in the other description. The need to generate this alternating pattern of slice types in each description prevents independent stream generation. That is, it is no longer possible to generate each description or decode it within its own control thread. Therefore, in this paper we apply redundant frames in a more direct manner that does not involve the need for a customized codec operation but does allow independently generated descriptions.

As also introduced in Section 1, FMO [19,38] is a promising form of video error resilience, which we use for single-path video transfer, adopting the type one checkerboard FMO pattern. By default in H.264/AVC, each frame forms a single slice group and macroblocks within that group are decoded in raster scan order. However, within a frame up to eight slice groups are possible. There are also seven different types of mapping between macroblock and slice group. Because type six supports arbitrary slice group mappings, its overhead is the greatest, as in addition to the slice group header a mapping must also be transmitted to the decoder (in a parameter set). Types three to five allow the size of each group to evolve over time, though macroblocks within a group remain geometrically contiguous. Only type one allows the assignment of geometrically dispersed macroblocks within a frame to form a slice group. The assignment is made through a mathematically function that in the two slice case results in a checkerboard

\footnotetext{
${ }^{2}$ It may also be possible to employ intra-coded macroblocks within B-frames in H.264/AVC, because unlike MPEG-4, this is now supported in H.264/AVC.
} 
pattern. Therefore, the overhead is lighter for this type. To reduce overhead, it is also preferable to choose the option in H.264/AVC that prevents reference outside the slice group, though at some cost in coding efficiency. A detailed analysis of overhead, which depends on encoder configuration, can be found in [19].

Significantly, the type one FMO checkerboard pattern is the only H.264 predefined mapping function that supports error concealment by interpolation of data from adjacent macroblocks in order to reconstruct missing macroblocks (if one of the two checkerboard slice packets were to be lost). Error concealment in H.264/AVC is a non-normative feature [35] in which the motion vectors of correctly received slices are computed if the average motion activity is sufficient (more than a quarter pixel). The recommendation [35] gives details of which motion vector to select to give the smoothest block transition. It is also possible to select the intra-coded frame method of spatial interpolation. In our FMO experiments, though experience favors a motion vector-based method, we employed both methods and selected the superior result in terms of average PSNR across the video sequence. In a live situation, it is possible to choose the method that best reduces 'blockiness' at macroblock boundaries. Notice that in non-FMO experiments, previous frame replacement was employed at the decoder to reconstruct a frame, as this is the normal form of error concealment for comparison purposes in such tests.

\section{Simulation model}

Simulation is the main tool for research on VANETs [34], because it is difficult to find an analytical solution due to the large number of variables involved such as vehicle density, speeds, and mobility patterns. It is also difficult to conduct repeated live experiments.

\subsection{Simulating IEEE $802.11 p$}

The Global Mobile System Simulator (GloMoSim) [45] simulation library was employed to generate our results. GloMoSim was developed based on a layered approach similar to the OSI seven-layer network architecture. Total simulation time was $900 \mathrm{~s}$, with the emergency video distribution starting after $100 \mathrm{~s}$. We employed IP framing with UDP transport, as TCP transport can introduce unbounded delay, which is not suitable for delay-intolerant video streaming. We simulated a multi-path variety of the Ad Hoc On demand Distance Vector (AODV) protocol but without strict enforcement of disjoint paths (either path or node disjoint) for the grid of Fig. 1. This allowed two paths to be selected for the MDC schemes. Though we are aware of considerable research in the field of multi-path protocol design, e.g. see [23] for multi-path video transport with a random waypoint mobility model, the intention of the current paper is to concentrate on video transport aspects. The BonnMotion mobility generator (http://web.informatik.unibonn.de/IV/Mitarbeiter/dewaal/BonnMotion/ accessed Sept. 2009) was chosen. Though BonnMotion does not model driver behaviour in the way that a mobility model such as VanetMobiSim [10] does, we considered it sufficient for generic simulations mainly intended to check the application's behavior. Besides, the driving behavior of emergency vehicles will be quite different from normal drivers.

A two-ray propagation model with an omni-directional antenna height of $1.5 \mathrm{~m}$ at receiver and transmitter was selected for which the reflection coefficient was -0.7 , which is the same as that of asphalt on tarmaced roads. The plane earth path loss exponent was set to 4.0, with the direct path exponent set for free space propagation (2.0). As in IEEE $802.11 \mathrm{p}$, transmission was at $5.9 \mathrm{GHz}$ with a bandwidth of $10 \mathrm{MHz}$. The transmission power was $33 \mathrm{dBm}(2 \mathrm{~W})$. The receiver power threshold was set to $-93 \mathrm{dBm}$, a normal value. Lastly, IEEE 802.11p's robust Binary Phase Shift Keying (BPSK) 
Table 1

Default simulation settings for Manhattan grid mobility model

\begin{tabular}{|c|c|}
\hline Parameter & Value \\
\hline Terrain dimension & $1000 \times 1000 \mathrm{~m}^{2}$ \\
\hline No. of vehicles & 100 \\
\hline Size of multicast group & 6 \\
\hline Number of $x-, y-b l o c k s$ & 10,10 \\
\hline Turn probability & 0.5 \\
\hline Speed change probability & 0.2 \\
\hline Minimum speed & $0.5 \mathrm{~m} / \mathrm{s}$ \\
\hline Average speed & $10.0 \mathrm{~m} / \mathrm{s}(22 \mathrm{mph})$ \\
\hline Speed standard deviation ${ }^{1}$ & $0.2 \mathrm{~m} / \mathrm{s}$ \\
\hline Speed update distance & $10 \mathrm{~m}$ \\
\hline Pause probability ${ }^{2}$ & 0 \\
\hline Transmission range & $150 \mathrm{~m}$ \\
\hline Routing protocol & AODV \\
\hline Wireless technology & IEEE $802.11 p$ \\
\hline Channel model & Two-ray \\
\hline
\end{tabular}

modulation mode was simulated, introducing a packet length dependency through Bit Error Rate (BER) modeling in a Additive Gaussian White Noise (AGWN) channel. Accordingly, the data-rate was set to 3 Mbps.

\subsection{Urban mobility model}

The essential features of an urban scenario are captured by mobility models. Two approaches are possible: either the detailed microcellular approach [34] or generic models [2]. The microcellular approach has the advantage that it includes the effect of obstacles such as lane closures, uphill gradients, and potholes. Though the generic models lack the detail of the microcellular approach, these models do allow systematic investigation and easily interpretable results. For the purposes of assessing how video streams can be effectively transferred, we have used a generic model that captures the essential feature of an urban scenario, the restricted mobility patterns imposed by the presence of city blocks.

In [2], two generic models relevant to vehicular mobility are described, namely Freeway Mobility and Manhattan Mobility. The Freeway model limits vehicles to 1-D motion in either direction. Vehicles are tied to one of several lanes; the speed is dependent on a vehicle's previous speed; and in the 'car-following' restriction, a following vehicle cannot exceed the speed of a preceding vehicle to avoid approaching within a safety distance. The Manhattan model, an extension of the Freeway model, restricts the number of lanes in either direction to just one, but introduces a turning probability to give greater mobility. Both Freeway and Manhattan are related in that they should result in high spatial and temporal dependency.

Default simulation settings for the Manhattan grid mobility model are given in Table 1, while individual simulations varied from the given defaults. In many urban settings it is likely that wireless-enabled cars would be restricted to average speeds of around $10 \mathrm{~m} / \mathrm{s}$ by congestion and traffic regulation, though vehicles responding to an emergency may go at faster speeds. The city block dimensions are chiefly related to the wireless range. Consequently, it is the relationship between range and block dimension that is important rather than the absolute settings. 


\subsection{Video configuration}

The reference Foreman video clip was encoded at QCIF resolution with 4:2:0 sampling. Foreman, intended for judging communication between mobile devices, exhibits the typical features of a hand-held camera and, because of camera pans, exhibits high to medium coding complexity. Each frame was generally coded as a single slice and encapsulated in an H.264/AVC Network Abstraction Layer unit (NALU) [39] before being placed in a single packet. The combination of RTP/UDP/IP headers results in a further $40 \mathrm{~B}$ of overhead. IDR-frames, however, were split into two slices, which reduces the peak data rate. If one of the IDR-frame packets arrives before another [37] partial decoding can still take place while the other packet arrives. The encoder was set to output in CBR mode, with initial quantization parameter of 32. The frame rate of the video stream was set at a slow scan rate of $15 \mathrm{~Hz}$ to avoid injecting too great a data-rate into the network. Consequently, for each stream in the MDC schemes the data rate was approximately $60 \mathrm{kbps}$.

For VRC, the skip frame(s) facility of H.264/AVC allowed the creation of even and odd frame sequences. For each sequence when using IDR frames, the Main profile of H.264 allowed B-frames to be included. The GOP size was 15 frames with the usual repeating pattern of two B- and one P-frame until the next IDR-frame. In the Main Profile, Context-Adaptive Binary Arithmetic Coding (CABAC) results in a 9-14\% bit saving at a small cost in computational complexity [24]. In the VRC variant and the redundant frame scheme, B-frames were no longer used. Intra-refresh macroblocks now provide the coding anchor points previously provided by IDR-frames. Intra-coded macroblocks were randomly selected (from a Uniform distribution) of H.264 macroblocks and were embedded with P-frames to the equivalent number needed for a QCIF IDR-frame. That is seven macroblocks per frame results in 105 macroblocks within a GOP, as opposed to $998 \times 8$ macroblocks for a single IDR-frame. Again the ability to generate random intra-coded macroblocks is a facility of H.264/AVC (H.264 JM Ref. Software, http://iphome.hhi.de/suehring/tml/download/, accessed Sept. 2009).

For FMO experiments, the Baseline Profile of H.264/AVC was selected with a GOP structure of IPP... In this Profile, intended for mobile devices and consequently with a smaller code footprint, Context Adaptive Variable Length Codes (dynamic Huffman entropic coding) is employed for simplicity (rather than CABAC), with some reduction in latency.

\section{Evaluation}

\subsection{Preliminary tests}

Figure 3 is a comparison between the luminance PSNR resulting from different error-resilient techniques upon Foreman, as the packet loss rate was varied. 100 simulation runs with different starting seeds were averaged to ensure convergence for Fig. 3. Errors in Fig. 3 followed a Uniform probability distribution function. Each frame was coded as a single slice, unless otherwise stated. Further detail of the error resilience methods compared is available in [31]. Notice that we have tested the methods separately for clarity, whereas a combination of methods in an error resilience strategy depending on channel conditions is possible, though there is a cost in extra overhead.

At around zero error packet loss-rate, FMO results in a somewhat lower video quality than omitting error resilience, because of its overhead, resulting in a lower coding efficiency. Separating into three independently-coded slices ('Slices' in Fig. 3), rather than one slice, is seen to be a little more effective at lower loss rates than in other loss regimes, as the risk of packet error is lower for shorter packet lengths. 


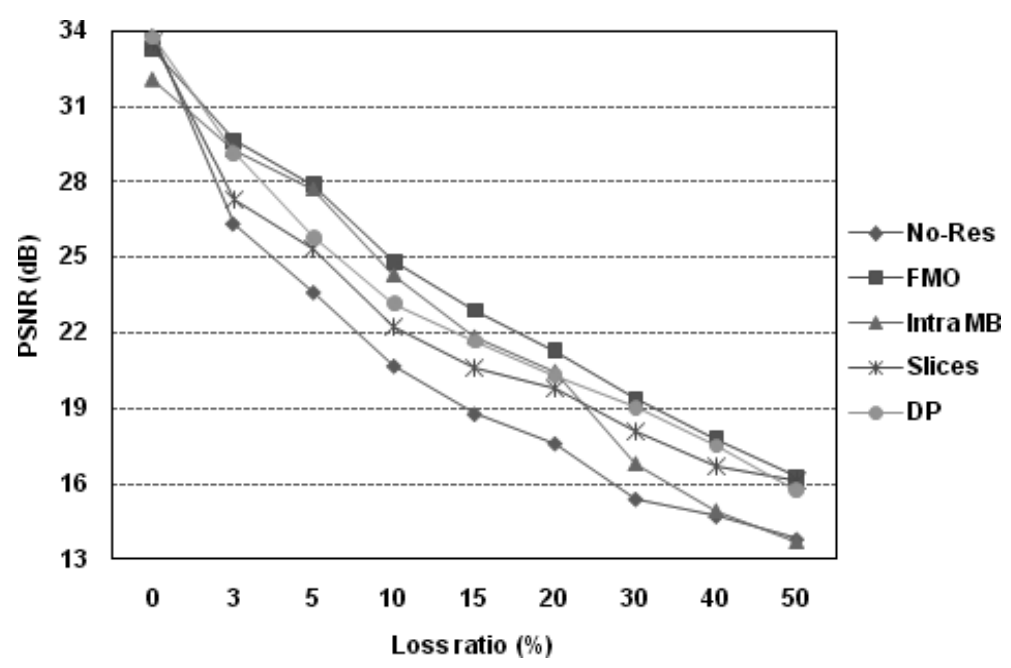

Fig. 3. Comparison between several H.264/AVC error resiliency methods and no resilience (No-Res) communication with Uniform bit errors, for Foreman QCIF sequence.

Data-partitioning ('DP' in Fig. 3 - separating configuration data and motion vectors, intra-coded, and inter-coded data into respectively A, B, and C NALUs) allows a frame to be effectively reconstructed even if the inter-coded macroblocks are missing, provided motion vectors in partition A are protected (which was simulated by assuming strong FEC protection of A NALUs). This technique is most useful at low loss rates. Insertion of Intra-coded Macroblocks ('Intra MB' in Fig. 3) allows temporal error propagation to be gradually arrested if some of a previous reference frame's macroblocks have been lost. In the tests, each row of macroblocks in rotation on a frame-by-frame basis was intra-coded. Intra-coded macroblocks in the tests was most helpful at medium loss rates. In the error conditions simulated, at medium to higher loss rates checkerboard FMO was the most effective method, though delivered video quality can no longer be considered fair for Foreman at a 10\% loss rate and higher. However, users normally accept quality at 25 to $30 \mathrm{~dB}$ if it is in a mobile application. As the vertical axis in Fig. 3 is effectively logarithmic, a gain of less than one $\mathrm{dB}$ will still make a difference at the boundary between a sequence being viewable or not viewable, though at PSNRs below $25 \mathrm{~dB}$ that gain is of little importance. Therefore, the main conclusion from Fig. 3 is that FMO is superior to other built-in error resilience methods of H.264/AVC except at very low error rates.

We also compared a selection of alternative FMO patterns, though for reasons of brevity and because it is not central to the main theme of this paper, the results are not plotted herein. The H.264/AVC Checkerboard pattern was superior to other built-in FMO patterns at higher loss rates, when coding QCIF sequences with two slices. At lower loss rates, checkerboard FMO is somewhat weaker but the quality is anyway good with whatever FMO pattern. FMO patterns tested were: a selection of foreground in one slice group and the remainder in another; row interleaving; raster scan ordering with two groups; and selection of columns or part columns. Therefore, the Checkerboard pattern should normally be selected for FMO, especially as it can be used for up to eight slice groups in H.264/AVC and is not confined to a two-slice scheme.

\subsection{Packet loss experiments}

Table 2 gives the packet size features of the protection schemes in the experiments. Packet sizes grow with the inclusion of intra-coded macroblocks and, because IDR-frames are split into at least two packets, 
Table 2

Protection schemes according to packet payload size

\begin{tabular}{lll}
\hline Scheme & Size range (B) & Characteristic \\
\hline VRC with IDR frames & 70 B to 885 B & $10 \%$ over 500 B \\
VRC with Intra-refresh & 183 B to 966 B & $50 \%$ over 450 B \\
Redundant frames multiple path & 260 B to 780 B & $10 \%$ over 500 B \\
FMO single path & 39 B to 493 B & $55 \%$ below 125 B \\
\hline
\end{tabular}

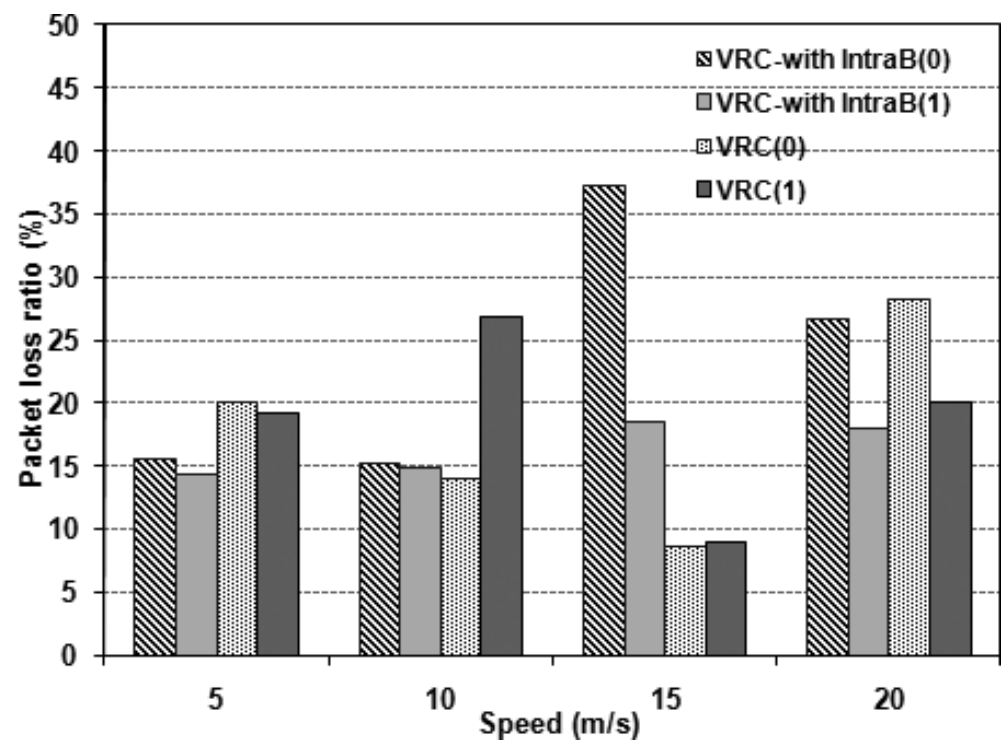

Fig. 4. Packet loss ratio for VRC for description (0) and description (1) versus vehicle speed with Table 1's settings, with IDR-frames and with intra-refresh macroblocks (marked IntraB).

larger packet sizes arise from VRC with intra-refresh than for VRC with IDR-frames. The smaller packet sizes for FMO are the result of splitting every frame into two slices, with a slice per NALU packet.

We firstly make some general remarks about the relationship between packet loss, vehicle speed and resulting video quality. As shown in Table 1, the number of emergency vehicle destinations is taken to be six, with one other video source vehicle, and the default total number of nodes is 100 . At a given speed, if vehicles are on average in proximity to each other for sufficient time for packet transfer then one can expect less packet loss to occur. However, a vehicle must also avoid travelling at too slow a speed, as then that vehicle may not have sufficient time to approach another vehicle to affect a further transfer. In general, our experiments showed packet loss rates to be difficult to predict and as Section 6.3 demonstrates, an unreliable indicator of video quality when different protection schemes are compared. There now follows experimental results using the three source-coding schemes.

\subsubsection{Packet loss under VRC}

Figure 4 shows that packet losses under multiple-path VRC whether with IDR-frames or with Intrarefresh are generally higher than $10 \%$. A level below about $10 \%$ is normally required for reasonable delivered video quality unless error protection is provided. There is only one speed $(15 \mathrm{~m} / \mathrm{s})$ at which packet loss is within bounds for the classical VRC scheme. Figure 5 shows the result of altering the number of vehicles available for multi-hop relay. There is a trend towards reduction of packet losses to an acceptable level as the network size is increased, because there are greater opportunities for packet 


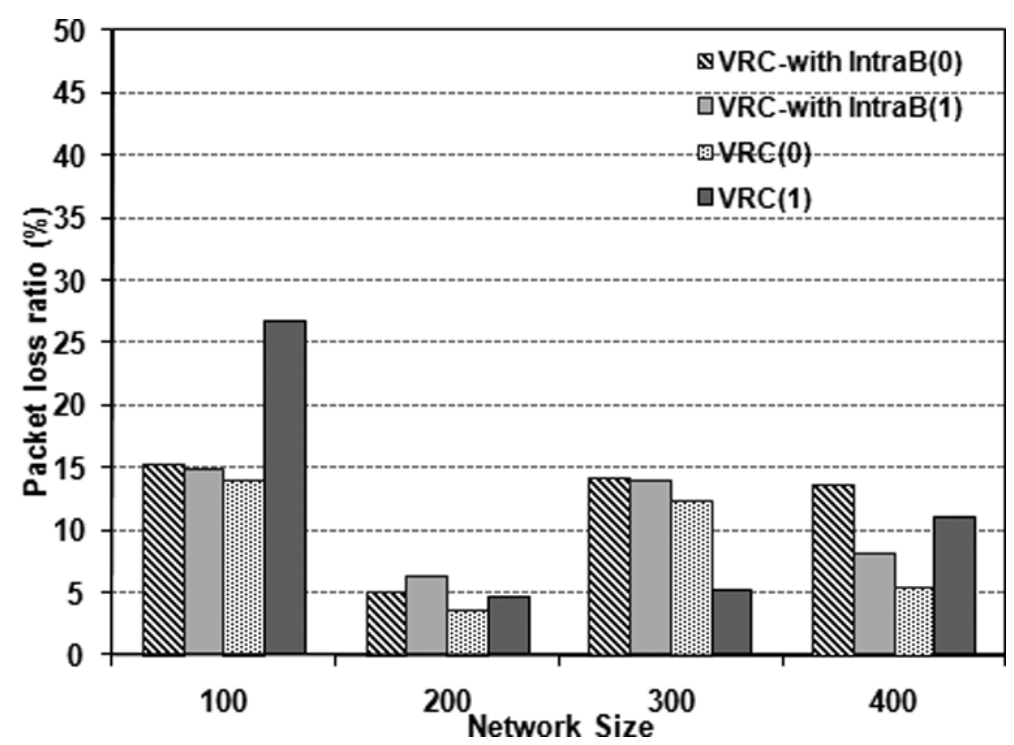

Fig. 5. Packet loss ratio for VRC for description (0) and description (1) versus network size with Table 1's settings, with IDR-frames and with intra-refresh macroblocks (marked IntraB).

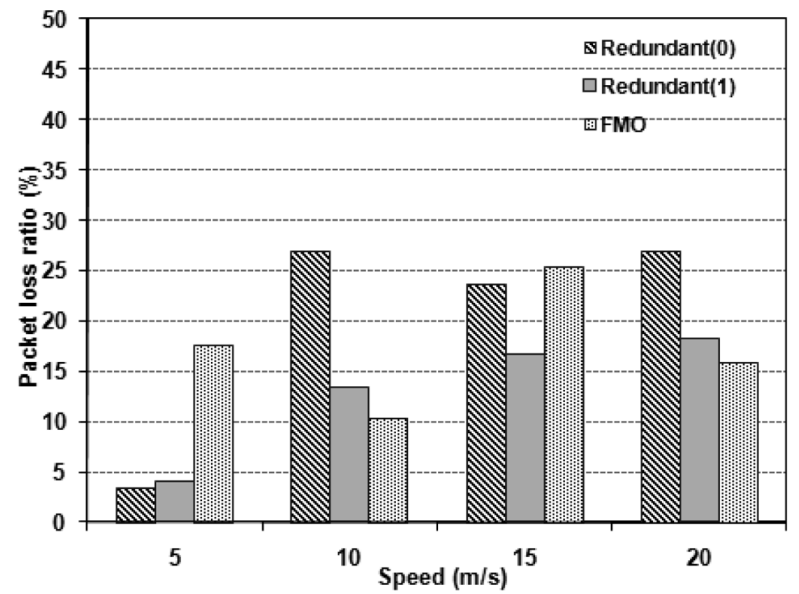

(a)

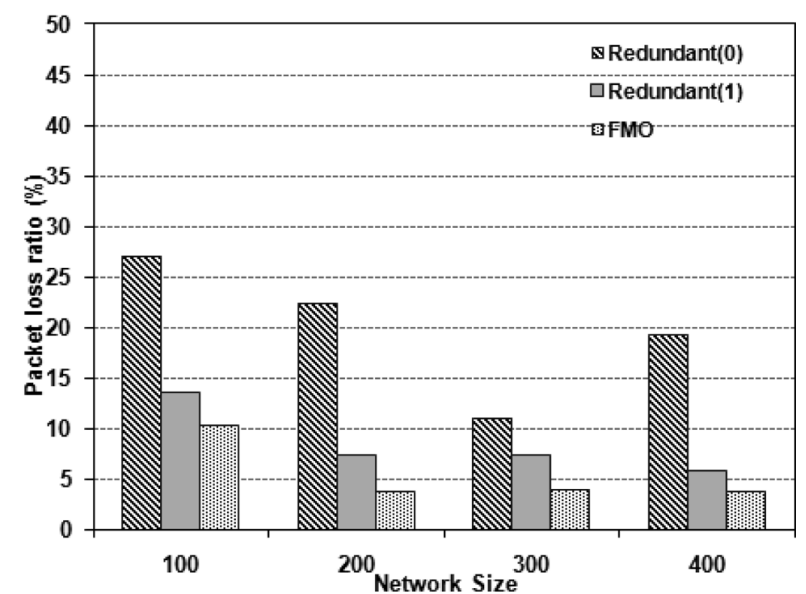

(b)

Fig. 6. Packet loss ratio for redundant frames and FMO with Table 1's settings with variation of a) vehicle speed and b) network size.

transfer to occur. In the experiment for IDR-frame VRC, each path might compensate for packet losses in the other path or description. Perhaps, because of the more regular data rate, this trend is not strongly apparent in the Intra-refresh version of VRC streaming. However, the main conclusion that can be drawn from these tests is that VRC in the form used herein is not satisfactory for video transport.

\subsubsection{Packet loss under Redundant Frames or FMO}

Figure 6 presents multiple-path video transfer packet loss when employing redundant frames. The packet loss levels with variation of speed or network size are generally higher than for VRC. However, packet loss rates are especially not a reliable guide to the likely video quality in this case, as, if redundant 
Table 3

Packet losses by protection scheme for the selected destination node

\begin{tabular}{ccccc}
\hline & \multicolumn{4}{c}{ Packet loss ratio (\%) } \\
\cline { 2 - 5 } & $\begin{array}{c}\text { VRC } \\
\text { (Intra-Refresh) }\end{array}$ & $\begin{array}{c}\text { VRC } \\
\text { (IDR-frames) }\end{array}$ & $\begin{array}{c}\text { Redundant } \\
\text { frames }\end{array}$ & FMO \\
\hline $\begin{array}{c}\text { Network size } \\
100\end{array}$ & 6 & 14 & 12 & 8 \\
200 & 1 & 2 & 15 & 3 \\
300 & 5 & 4 & 3 & 4 \\
400 & 4 & 6 & 10 & 2 \\
Speed & & 3 & 1 & 15 \\
5 & 2 & 14 & 12 & 8 \\
10 & 6 & 6 & 10 & 25 \\
15 & 18 & 9 & 15 & 13 \\
$\mathbf{2 0}$ & 8 & & &
\end{tabular}

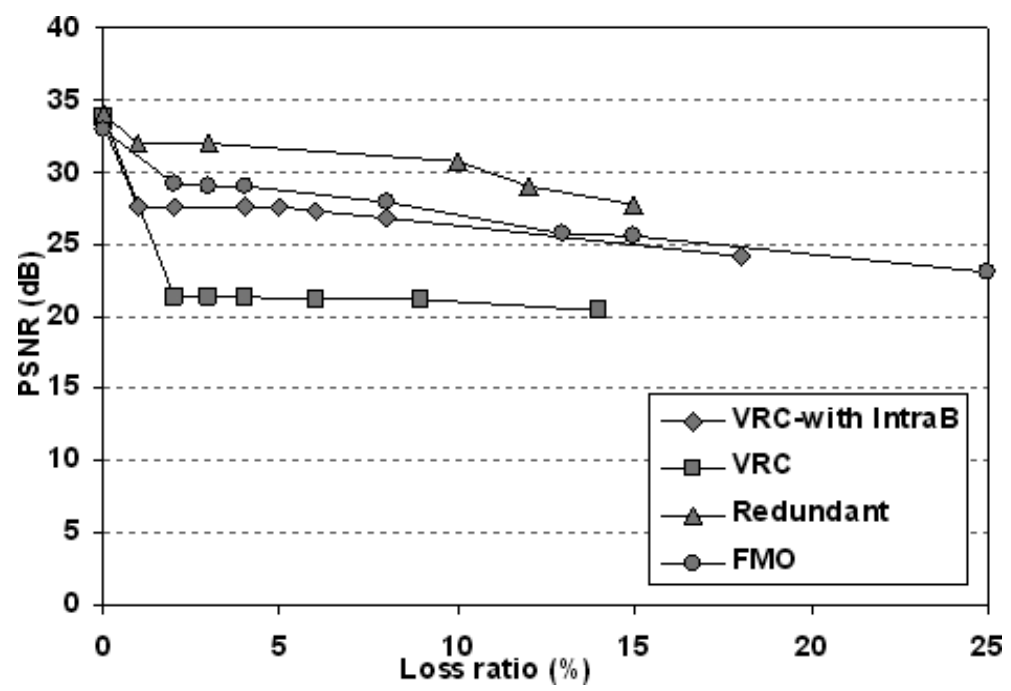

Fig. 7. Video quality by protection scheme for the selected node according to the packet loss ratios of Table 3 . VRC $=$ VRC with IDR frames, Redundant $=$ Multiple path with redundant frames, VRC with IntraB $=$ VRC with Intra-refresh macroblocks

frame packets are dropped, the video quality remains the same. Of course, a mixture of redundant and active frame packet loss will actually occur. Turning to single-path FMO error resilience, from Fig. 6 also, there is generally a much reduced level of packet loss for larger network sizes at the default speed, but FMO packet loss is sensitive to vehicle speed. Therefore, the main conclusions are that redundant frames lead to larger packet losses, though this may not necessarily result in worse video quality, whereas single-path FMO suffers less packet loss.

\subsection{Video performance}

From the six destination nodes, a 'median' node was selected in terms of packet loss, rather than one experiencing very high or very low packet loss. Table 3 reports the packet loss statistics for the chosen node. There is a decreasing trend in packet losses with network size, but no clear trends are apparent in terms of vehicle speed. 

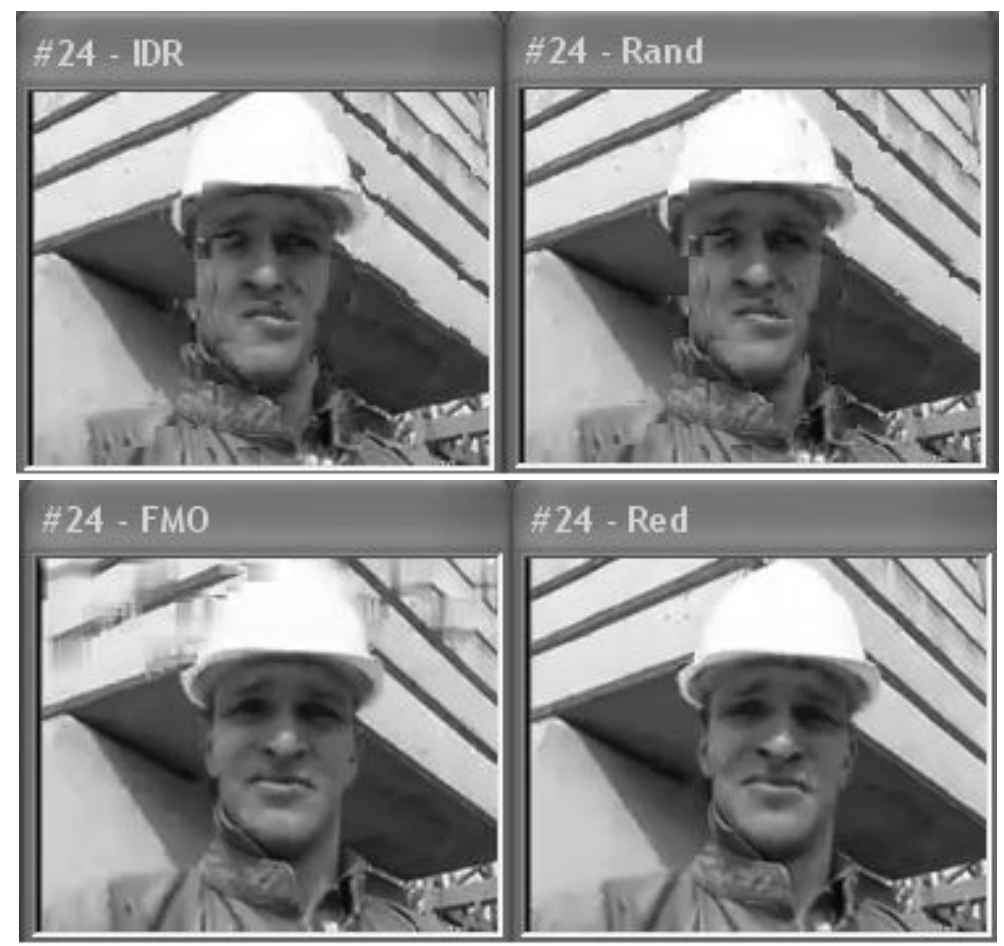

Fig. 8. Sample equivalent frames from the protection schemes. IDR $=$ VRC with IDR frames, Rand $=$ VRC with randomised Intra-refresh, Red $=$ Redundant frames with multiple path

\subsubsection{Video quality}

Figure 7 compares resulting delivered video quality (PSNR) at the default settings. Recall that for PSNR the vertical scale is logarithmic. Employing redundant frames, despite the comparatively high packet loss ratios, results in good video quality. On average, with the packet loss ratios found from the simulation, sufficient redundant frames survive to repair the composite video frame sequence. FMO allows single stream video transfer to compete with multiple-path transfer but video quality is only fair in some settings tested. Including Intra-refresh macroblocks does improve the PSNR of VCR but not sufficiently to compete with the redundant frame scheme. Therefore, the main conclusion is that both redundant frames and FMO achieve reasonable video quality in the given scenario.

For illustrative purposes only, a sample frame in the Foreman sequence is shown in Fig. 8 for each of the schemes. In the two VRC schemes, there is considerable distortion around the face as previous frame error concealment has been affected by motion of the face between the frames. FMO results in less distortion but the cream-slated background shows some distortions.

\subsubsection{Video latency and control overhead}

Moreover, in broad terms from Fig. 9, average end-to-end delay (for all vehicles) is longer in duration for the VRC schemes. Generally, across all schemes packet delay times are significant and do require buffering. For other applications, e.g. a Web click and view service, there would be a perceptible start-up delay between selecting the video and starting to view the stream. For the emergency scenario, the destination emergency vehicles are unaware of the start time of the stream at the source and, therefore, would not notice the small delay. However, the time available to view the scene as a vehicle approached 


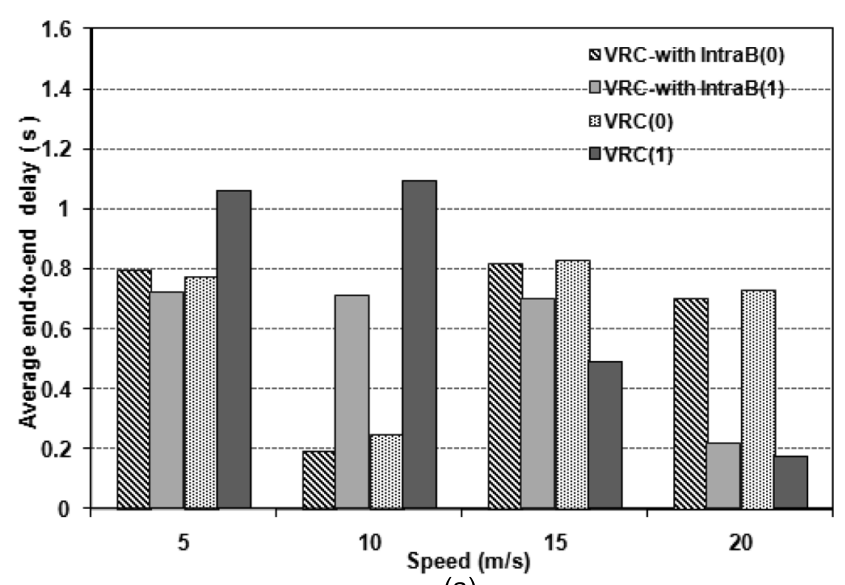

(a)

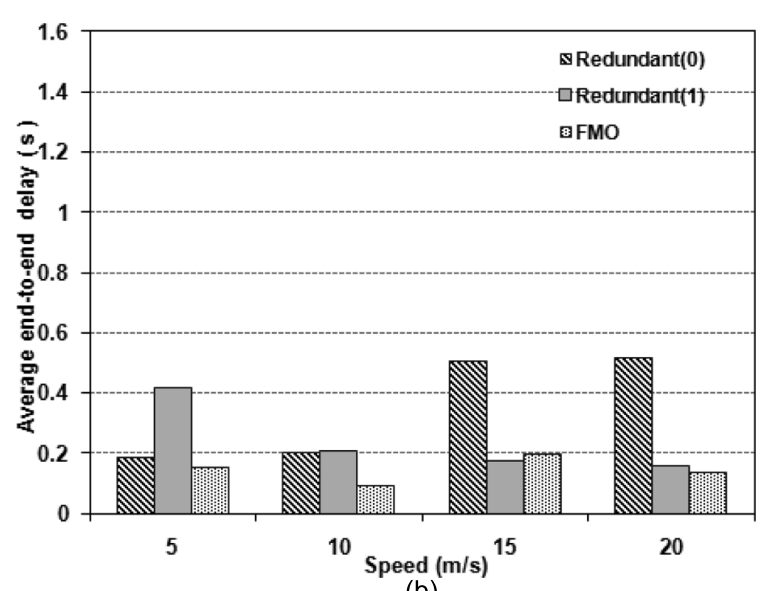

(b)

Fig. 9. End-to-end packet delay according to vehicle speed for a) VRC schemes. b) non-VRC schemes.

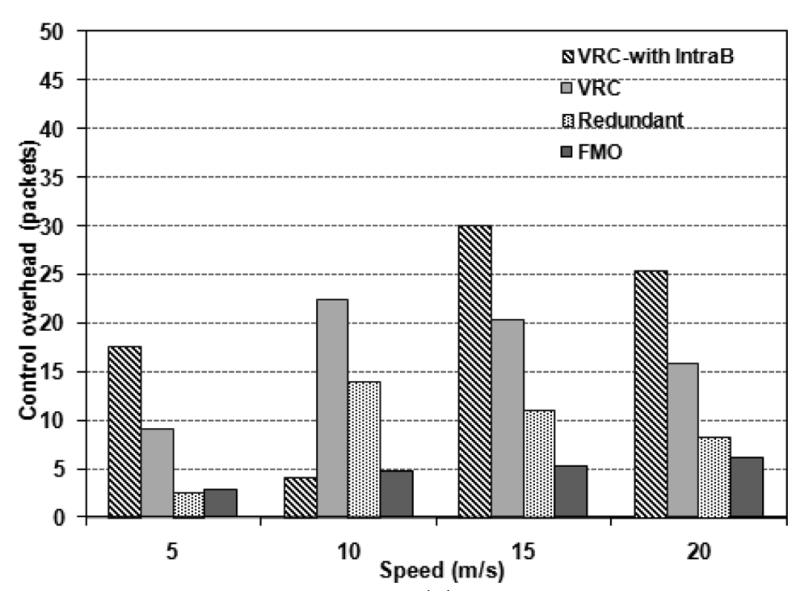

(a)

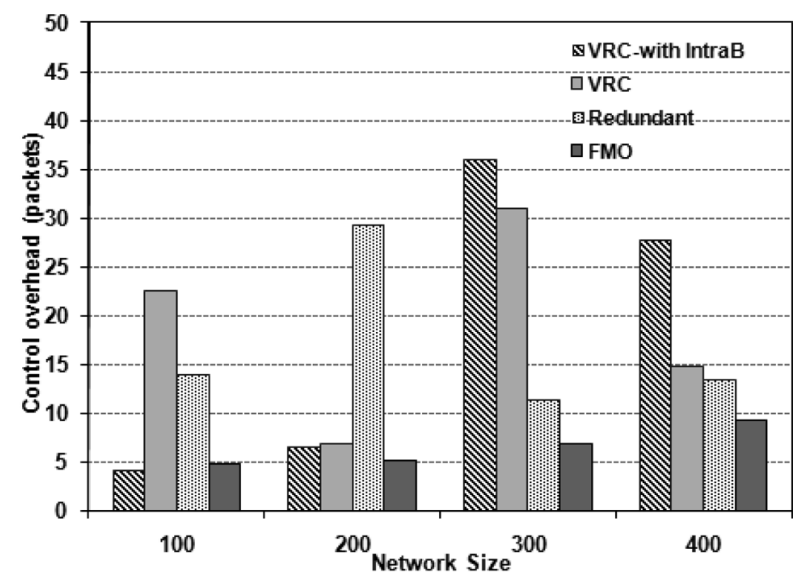

(b)

Fig. 10. Control packet overhead according to a) vehicle speed b) network size.

the emergency site would be reduced. The end-to-delay levels for VRC schemes were found to be similar to those for the FMO and redundant frames schemes when the network size was greater then 100 nodes. Therefore, Fig. 9a represents a worst case for the VRC schemes. Jitter was found to be broadly similar across the schemes and scenarios, with a range from 0.04 to $0.07 \mathrm{~s}$. At 15 frame/s, this represents a need for a two-frame jitter buffer.

From Fig. 10, the number of control overhead packets was least for FMO, because only one stream was transmitted, whereas especially when speed was varied the redundant frame scheme resulted in reduced control packet overhead compared to the VRC schemes. However, the number of control packets expended to deliver a packet can be high. Moreover control packets add to the overall congestion in a network even though within a VANET the extra energy consumed in transmitting them may be discounted (as a vehicle's alternator is a convenient power source). This might be alleviated by employing a different multi-path routing protocol, e.g. [20], to route over multiple paths. However, this aspect is left to future work as the authors are aware that there are many issues involved in choice of protocol.

Therefore, combining the impact of extra latency and control overhead implies that a compromise 
solution is to use FMO rather than redundant frames fro transmitting video streams in this scenario. In our tests, VRC is not only weaker in terms of delivered video quality but it increases latency and overhead.

\section{Tentative analytical model}

This Section constructs the components of a tentative analytical model that aims to provide a way of calculating delivered video quality. The long term delivered video quality depends on the connectivity of the network, as this determines the ability of vehicles to communicate across the VANET. In turn, the speed of the vehicles is required to determine the connectivity. For example, the speed of vehicle $i$ at time $t, \nu_{i}(t)$, in the Manhattan grid model [2] of the simulations is given by:

$$
\begin{aligned}
\nu_{i}(t+\Delta t) & =\nu_{i+1}(t)-a / 2, \text { if } \Delta x_{i}(t) \leqslant \Delta x_{\min } \\
& =\tilde{\nu}_{i}(t+\Delta t), \text { otherwise } \\
\tilde{\nu}_{i}(t+\Delta t) & =\min \left(\max \left(\nu_{i}(t)+\eta a \Delta t, \nu_{\min }\right), \nu_{\max }\right)
\end{aligned}
$$

where $\Delta x_{i}(t)$ is the bumper-to-bumper distance from vehicle $i$ to the vehicle in front, $\Delta x_{\min }$ is the minimum safety distance, $a$ is the vehicle acceleration, and $\eta$ is a uniformly distributed random variable in the range $[0,1]$. Notice that unlike the well-known Random Waypoint model, in the Manhattan grid model the motion of a vehicle is dependent on its previous motion and on the position of the car in front. Other micro-cellular motion models can be found in [11]. As observed from the simulations in Section 6, node density has an important role. In order to incorporate node density into a stochastic model, the mathematically-tractable Poisson arrival process can be employed [18] to model vehicle arrivals along roads leading into the terrain (the Manhattan grid road layout). It is not claimed that a Poisson process is a model for the vehicle arrival process but use of this process does provide a point of comparison. The traffic intensity $\lambda$ is then given by

$$
\lambda=\frac{2 f}{\nu_{\text {mean }}}
$$

where $\nu_{\text {mean }}$ is the average speed of vehicles with a Poisson arrival process of intensity $f$ in terms of vehicles/hour/road, with two lanes per road assumed in Eq. (3). In a Manhattan grid of size $\mathrm{N} \times \mathrm{N}$ blocks, there are $4(\mathrm{~N}-1)$ roads for vehicles to enter or leave the terrain. In [18], for a grid with $\mathrm{N}=10$, a road segment length of $400 \mathrm{~m}$ (100 m segments were used in our simulations), with a car-following driver model (dependent on the car in front with some modelling of driver behaviour [11]), it was found that intensity saturated at around $f=800$ to 1000 vehicles/h/lane.

Assuming all vehicles are wireless-enabled, then wireless communication essentially depends on the range $r$. If there are some cars that are not wireless enabled then a factor $\rho$ in the range $[0,1]$ is introduced. The factor $\rho$ also affects traffic congestion and in that way connectivity. However, in the simulations of this paper $\rho$ is effectively set to unity. In steady-state, i.e. the number of vehicles leaving and entering the terrain is balanced, an upper bound on the expected number of vehicles that are not connected to any other vehicle, $n$, is:

$$
E[n]=\exp (-2 \lambda \rho r)
$$


In the aforementioned setting [18], Eq. (4) was found to give a reasonable fit to simulated results for values of $\lambda$ up to about 15 vehicles $/ \mathrm{km}$. However, this was for linear motion across the terrain, i.e. entering at one end of a road and leaving at the other end, and not to random destinations, as in this paper's results.

The range is governed by the propagation model. Thus, for the two-ray ground reflection path loss model used in our simulations, if the distance $d$ is more than the cross-over distance [27] then the received power is:

$$
P_{r}(d)=\frac{P_{t} g_{t} g_{r} h_{t}^{2} h_{r}^{2}}{d^{4} L}
$$

where $g_{t}$ and $g_{r}$ are the gains of the transmitter and receiver respectively, $h_{t}$ and $h_{r}$ are the antenna heights of the transmitter and receiver respectively, $P_{t}$ is the power at the transmitter, and $L$ is the system loss. For distances less than the cross-over distance, the Friis free-space model [27] is applied, namely:

$$
P_{r}(d)=\frac{P_{t} g_{t} g_{r} \Lambda^{2}}{(4 \pi)^{2} d^{2} L}
$$

where $\Lambda$ is the wavelength. If power thresholding is applied at the receiver then this model allows a simple prediction of range. However, in the simulations packet length modelling was performed according to the form of the modulation for an AGWN channel [27]. The cross-over distance, $d_{c}$, is:

$$
d_{c}=\left(4 \pi h_{t} h_{r}\right) / \Lambda
$$

Assuming some function, $g$, can be found that relates connectivity to packet loss then, from experience with the error-resilience techniques in the paper, these techniques act to additively increase the PSNR. For example, a $10 \%$ packet loss rate may achieve overall a fair video quality of $25 \mathrm{~dB}$ PSNR or above. However, if the packet loss rate is more that $10 \%$, then error-resilience can restore the quality for excess (over 10\%) packet loss rates up to total packet loss rate of about $20 \%$. Thus, the effective packet loss rate is given by:

$$
R_{e f f}=g(E[n])-C
$$

where $C$ is the reduction in packet loss rate due to error resilience (say $10 \%$ ). If $R_{e f f}$ is greater than a given threshold value (say) $10 \%$ then video quality is judged to be unacceptable for all vehicles in the VANET multicast group. This simple model may be adjusted according to the number of paths employed and the strength factor of the error-resilience method. However, further development of this model is beyond the scope of the present paper.

\section{Conclusions}

This paper has investigated whether the operating conditions in a city are likely to permit video communication. It has found that MDC with redundant frame insertion (a new suggestion for VANETs) and single stream FMO, both source coding techniques included in the H.264/AVC codec, can support robust communication when packet loss rates are relatively high. Video communication to a group of emergency vehicles allows recognition of suspect vehicles, description of burning buildings and the like to be passed from the first vehicle on the scene to approaching vehicles. End-to-end delay remains a 
concern which should be addressed by reduction of hop counts by the routing protocol. As this is an outdoor scenario, location aware routing based on the global positioning system will be investigated. Further work will also consist of detailed investigation of urban wireless propagation conditions and modelling of driver behaviour.

\section{References}

[1] J. Apostolopoulos, Reliable video communication over lossy packet networks using multiple state encoding and path diversity, Visual Comms: Image Processing (Jan 2001), 392-409.

[2] F. Bai, N. Adagopan and A. Helmy, IMPORTANT: A framework to systematically analyze the Impact of Mobility on Performance of routing protocols over Ad hoc NeTworks, IEEE INFOCOM (April 2003), 825-835.

[3] S. Biswas, R. Tatchiko and F. Dion, Vehicle-to-vehicle wireless communication protocols for enhancing highway traffic safety, IEEE Communications Mag 44(1) (Jan 2007), 74-82.

[4] J.J. Blum, A. Eskandarian and L.J. Hoffman, Challenges of inter-vehicle ad hoc networks, IEEE Trans. on Intelligent Transportation Systems 5(4) (2004), 347-351.

[5] P. Bucciol, E. Masala, N. Kawaguchi, K. Takeda and J.C. de Martin, Performance evaluation of H.264 video streaming over inter-vehicular 802.11 ad hoc networks, IEEE 16th Int Symp on Personal, Indoor and Mobile Radio Comms (Sept 2005), 1936-1940.

[6] N. Chand, R.C. Joshi and M. Misra, Cooperative caching in mobile ad hoc networks based on data utility, Mobile Information Systems 3(1) (2006), 19-37.

[7] Y.C. Chang, M.S. Beg and T.S. Tang, Performance evaluation of MPEG-4 video error resilient tools over a mobile channel, IEEE Trans on Consumer Electronics 49(1) (Feb 2003), 6-13.

[8] S.R. Dickey, C.-L. Huang and X. Guan, Field measurements of vehicle to roadside communication performance, IEEE Vehicular Technol Conf (Fall 2007), 2179-2183.

[9] J. Dunlop, D. Girma and J. Irvine, Digital Mobile Communications and the TETRA System, J. Wiley \& Sons, Chichester, UK, 2000.

[10] M. Fiore, J. Härri, F. Filali and C. Bonnet, Vehicular mobility simulation for VANETs, 40th Annual Simulation Symposium (Mar 2007), 301-307.

[11] M. Fiore and J. Härri, The network shape of vehicle mobility, 9th ACM Int'l Symposium on Mobile Ad Hoc Networking and Computing (2008), 261-272.

[12] M. Gerla, R.G. Cascella, Z. Cao, B. Crispo and R. Battiti, An efficient weak secrecy scheme for network coding data dissemination in VANET, IEEE PIMRC (Sept 2008), 1-15.

[13] M. Ghanbari, Standard codecs: Image compression to advanced video coding, IET Press, London, UK, 2003.

[14] V.K. Goyal, Multiple description coding: Compression meets the network, IEEE Signal Process Mag 18(5) (Sept 2001), 74-93.

[15] M. Guo, M.H. Ammar and E.W. Zegura, V3: A vehicle-to-vehicle live video streaming architecture, 3rd IEEE Int'l Conf. on Pervasive Computing and Comms (Mar 2005), 171-180.

[16] A.M. Hanashi, I. Awan and M. Woodward, Performance evaluation with different mobility models for dynamic probabilistic flooding in MANETs, Mobile Information Systems 5(1) (2009), 65-80.

[17] D. Jiang and L. Delgrossi, IEEE 802.11p: Towards an international standard for wireless access in vehicular environments, IEEE Vehicular Technol Conf (May 2008), 2036-2040.

[18] M. Kafsi, P. Papadimitratos, O. Dousse, T. Alpcan and J.-P. Hubaux, VANET connectivity analysis, IEEE Workshop on Automotive Networking and Applications (Dec 2008).

[19] P. Lambert, W. Deneve, Y. Dhondt and R. Vandewalle, Flexible macroblock ordering in H.264/AVC, J of Visual Communication and Image Representation 17 (2006), 358-375.

[20] S.-J. Lee and M. Gerla, Split multipath routing with maximally disjoint paths in ad hoc networks, IEEE Int'l Conf. on Communications (Jun 2001), 3201-3205.

[21] H.V. Leong and A. Si, Multi-resolution information transmission in mobile environments, Mobile Information Systems 1(1) (2005), 25-40.

[22] L. Lima, M. Médard and J. Barros, Random linear network coding: A free cipher? IEEE Int'l Symp on Info Theory (Jun 2007).

[23] Q. Lu, L. Du, Z. Zuo and X. Xiao, Improved multi-path AODV protocols for real-time video transport over Mobile Ad Hoc Networks, IEEE Pacific-Asia Workshop on Computational Intelligence and Industrial Application (2008), 621-625.

[24] D. Marpe, H. Schwarz and T. Wiegand, Context-based adaptive binary arithmetic coding in the H.264/AVC video compression standard, IEEE Trans on Circuits and Systems for Video Technol 13(7) (2003), 620-636. 
[25] J.S. Park, L. Uichin, S.Y. Oh, M. Gerla and D. S. Lun, Emergency related video streaming in VANET using network coding, 3rd International Workshop on Vehicular Ad Hoc Networks (2006), 102-103.

[26] I. Radulovic, Y.-K. Wang, S. Wenger, A. Hallapuro, M.H. Hannuksela and P. Frossard, Multiple description H.264 video coding with redundant pictures, Int'l Workshop on Mobile Video (Sept 2007), 37-42.

[27] T.S. Rappaport, Wireless Communications, (2nd edition), Prentice-Hall, Upper Saddle River, NJ, 20012.

[28] M. Roccetti, M. Gerla, C.E. Palazzi, S. Ferretti and G. Pau, First responders' crystal ball: How to scry the emergency from a remote vehicle, IEEE 26th Int'l Conf on Performance of Computing and Communs (April 2007), 556-556.

[29] S. Saponara, C. Blanch, K. Denolf and J. Bormans, The JVT advanced video coding standard: Complexity and performance analysis on a tool-by-tool basis, Int'l Packet Video Workshop (April 2003).

[30] R.M. Schreier and A. Rothermel, Motion adaptive intra refresh for the H.264 video coding standard, IEEE Trans. on Consumer Electronics 52(1) (2006), 249-253.

[31] T. Stockhammer and W. Zia, Error-resilient coding and decoding strategies for video communication, in: Multimedia over IP and Wireless Networks, M. van der Schaar and P.A. Chou, eds, Academic Press, Amsterdam, 2007, pp. 59-80.

[32] D. Tian, M.M. Hannuksela, Y.-K. Wang and M. Gabbouj, Error resilience coding techniques using spare pictures, Int'l Packet Video Workshop (April 2003).

[33] T. Tillo, M. Grangetto and G. Olmo, Redundant slice optimal allocation for H.264 multiple description coding, IEEE Trans Circuits and Syst for Video Technol 18(1) (2008), 59-70.

[34] O.K. Tonguz, W. Viriyasitavat and F. Bai, Modeling urban traffic: A Cellular automata approach, IEEE Communications Mag 47(5) (May 2009), 142-150.

[35] V. Varsa, M.N. Hannuksela and Y. Wang, Non-normative error concealment algorithms, ITU-T SGI6 Doc., VCEG-N62, 2001.

[36] Y. Wang, A.R. Reibman and S. Lee, Multiple description coding for video delivery, Proc of the IEEE 93(1) (2005), $57-70$.

[37] W. Wei and A. Zakhor, Multipath unicast and multicast video communication over wireless ad hoc networks, Int'l Conf on Broadband Networks (Oct 2004), 494-505.

[38] S. Wenger and M. Horowitz, Flexible MB ordering - a new error resilience tool for IP-based video, Int'l Workshop on Digital Communications, Capri, Italy, (2002).

[39] S. Wenger, H264/AVC over IP, IEEE Trans Circuits and Syst for Video Technol 13(7) (2003), 645-656.

[40] S. Wenger, G.D. Knorr, J. Ou and F. Kossentini, Error resilience support in H.263+, IEEE Trans Circuits and Syst for Video Technol 8(7), 867-877.

[41] T. Wiegand, G.J. Sullivan, G. Bjøntegaard and A. Luthra, Overview of the H.264/AVC video coding standard, IEEE Trans Circuits and Syst for Video Technol 13(7) (July 2003), 560-576.

[42] Z. Wu and J.M. Boyce, Adaptive error resilient video coding based on redundant slices of H.264/AVC, IEEE Int'l Conf. on Multimedia and Expo (July 2007), 2138-2141.

[43] S. Yousefi, M.S. Mousavi and M. Fathy, Vehicular ad hoc networks (VANETS): Challenges and perspectives, 6th Int'l Conf on ITS Telecommunications (Jan 2006), 761-766.

[44] H. Yu, Streaming media encryption, in: Multimedia Security, B. Furht and D. Kirovski, eds, CRC Press, Boca Raton, FO, 2005, pp. 197-220.

[45] X. Zeng, R. Bagrodia and M. Gerla, GloMoSim: A library for parallel simulation of large-scale wireless networks, 12th Workshop on Parallel and Distributed Simulations (May 1998).

Nadia N. Qadri received her $\mathrm{PhD}$ at the School of Computer Science and Electronics Engineering, University of Essex, UK in 2010. She received her Masters of Engineering (Communication Systems and Networks) and Bachelors of Engineering (Computer Systems), from Mehran University of Engineering and Technology, Jamshoro, Pakistan in 2004 and 2002 respectively. She has more than four years of teaching and research experience at renowned universities of Pakistan viz. Mehran University of Engineering \& Technology, Fatima Jinnah Women's University and COMSATS Institute of Information Technology. Her research interests include video streaming for mobile ad hoc networks and vehicular ad hoc networks, along with P2P streaming.

Muhammad Altaf received his BSc degree from the University of Engineering and Technology, Peshawar, Pakistan in 2001 and his MSc degree in computer system engineering from the National University of Science and Technology, Rawalpindi, Pakistan in 2004. He has recently been awarded his PhD at the University of Essex, UK. His research interests are video compression and video streaming over wired and wireless networks.

Martin Fleury has a degree in Modern History (Oxford University, UK) and a Maths/Physics based degree from the Open University, Milton Keynes, UK. He obtained an MSc in Astrophysics from QMW College, University of London, UK in 1990 and an MSc from the University of South-West England, Bristol in Parallel Computing Systems in 1991. He holds a PhD in Parallel Image Processing Systems from the University of Essex, Colchester, UK. He is currently employed as a Senior 
Lecturer at the University of Essex. Martin has authored over 160 articles, and other publications on the subjects of low-level image- and signal-processing algorithms (including document and image compression algorithms), performance prediction of parallel systems, software engineering, and vision systems. His current research interests are video communication over MANS, WLANs, PANs, BANs, MANETs, and VANETs.

Mohammed Ghanbari is best known for his pioneering work on two-layer video coding for ATM networks (which earned him an IEEE Fellowship in 2001), now known as SNR scalability in the standard video codecs. He has served as an Associate Editor for IEEE Trans. on Multimedia. He has registered for eleven international patents on various aspects of video networking and was the co-recipient of A.H. Reeves prize for the best paper published in the 1995 Proc. of IEE on the theme of digital coding. $\mathrm{He}$ is the co-author of "Principles of Performance Engineering", a book published by IET press in 1997, the author of "Video Coding: An Introduction to Standard Codecs", a book also published by IET press in 1999, which received the year 2000 best book award by the IEE, and the author of "Standard Codecs: Image Compression to Advanced Video Coding" also published by the IET press in 2003. Prof. Ghanbari has authored or co-authored about 450 journal and conference papers, many of which have had a fundamental influence in this field. 

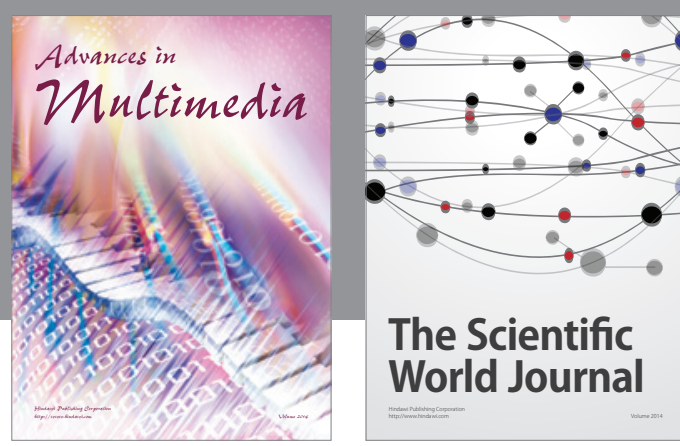

The Scientific World Journal
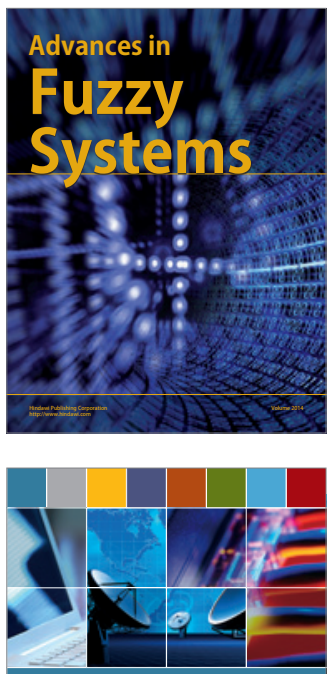

Computer Networks and Communications
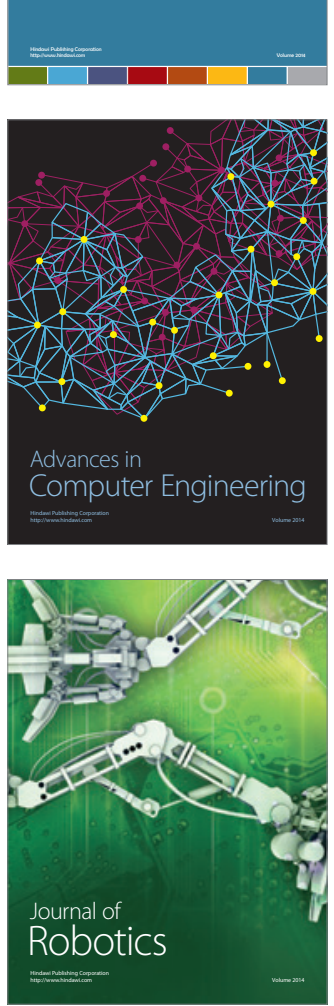
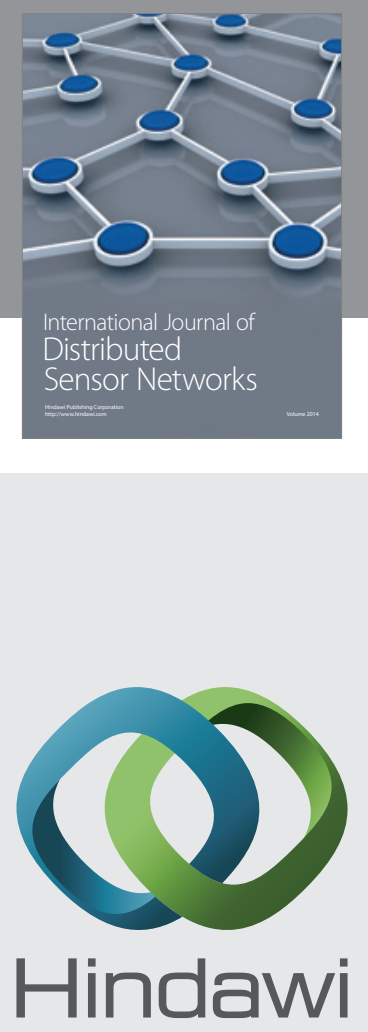

Submit your manuscripts at

http://www.hindawi.com
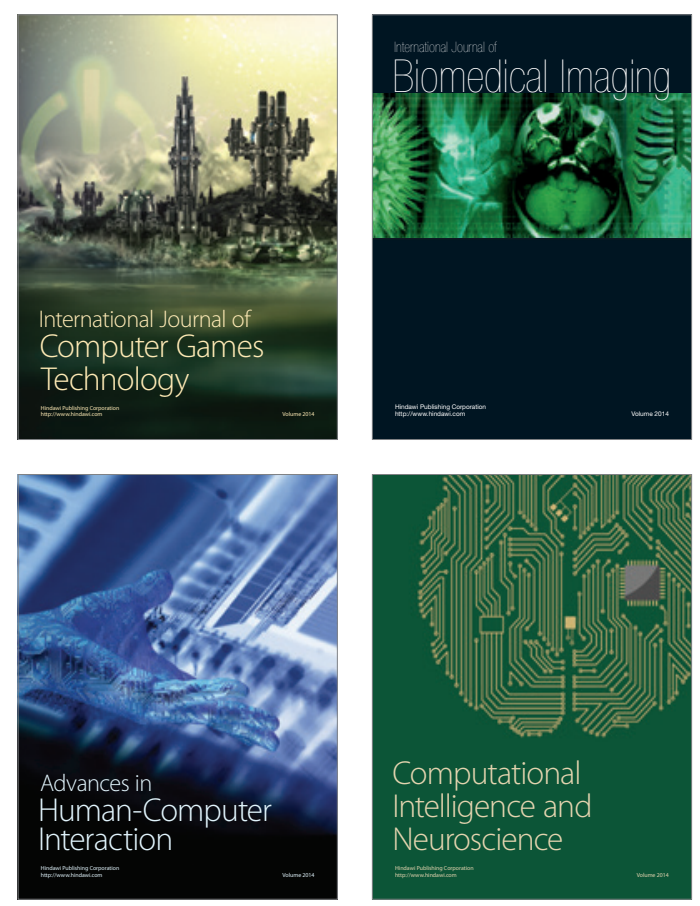
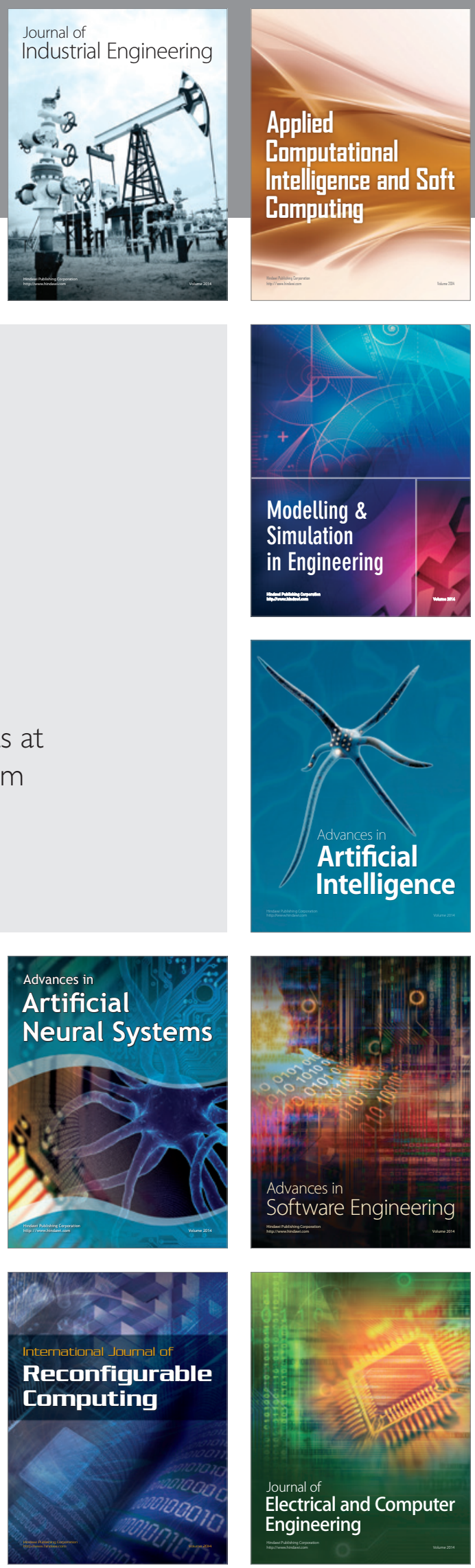